\title{
The European Factor: From ARM to Atos
}

\author{
Anne C. Elster ${ }^{1}$ \\ ${ }^{1}$ Affiliation not available
}

December 7, 2020

It has been both a strange and unsettling year with a lot of uncertainties related to COVID-19. Both the premier supercomputing conferences ISC'20 and SC20 went virtual, preventing users from exploring the show floors with their examples of novel architectures and seeing the demos of their related tools. Companies have also slowed their procurement of high-end systems during 2020, as was discussed during the presentation of top500.org statistics at the Top-500 Birds-of-a-Feather session at SC20. The Top-500 list is a measurement of the world's largest computers using the High-Performance Linpack benchmark (see www.top500.org). Systems must solve large dense systems of linear equations without using the operation-saving Strassen algorithm or a mix of lower precision, but doing the calculations conforming to LU factorization with partial pivoting using $2 / 3 n^{3}+O\left(n^{2}\right)$ double-precision (64-bit) floating point numbers.

Despite less turnover of the world's largest computer systems, technologies continue to advance, and the trends are more interesting and diverse than ever.

The US dominated the computing industry - including the supercomputer industry - for years, until Japan gave the US a jolt with the introduction of the Earth Simulator back in 2002. This put supercomputing back in the spotlight, especially in the US, but also in Europe. For the past decade, the US, Japan and China have dominated at the top of the Top-500 list. As can be seen from the following table, these systems were built primarily on US-designed chips from IBM and Intel, coupled with accelerators from NVIDIA. The AMD-based Titan Cray/HPE system and the Sunway system in China, as well as the new ARM-based Fujitsu Fugaku supercomputer, are notable exceptions. In this article, we will discuss the history of ARM and its current impact on HPC as well as some other European trends. 


\begin{tabular}{|c|c|c|c|c|}
\hline Name & Dates no. 1 & Where & Architecture & Cores \\
\hline $\begin{array}{l}\text { Tianhe- } \\
\text { 1A }\end{array}$ & 2010-11-01 & China & $\begin{array}{l}\text { NUDT TH MPP, Intel Xeon X5670 @ } 2.93 \text { GHz, } \\
\text { Infiniband FDR FT-1000, Nvidia } 2050 \text { GPUs }\end{array}$ & 186368 \\
\hline $\begin{array}{c}\text { K- } \\
\text { Computer }\end{array}$ & $\begin{array}{l}\text { June } 2011- \\
\text { Nov } 2011\end{array}$ & Japan & Fujitsu SPARC64 VIIIfx @ 2.0GHz with Tofu interconn. & 705024 \\
\hline Sequoia & $2012-06-01$ & US & $\begin{array}{l}\text { IBM BlueGene/Q, IBM Power BQC 16C @ } 1.6 \mathrm{GHz} \text {, } \\
\text { Custom interconnect }\end{array}$ & $\begin{array}{c}1572 \\
864\end{array}$ \\
\hline Titan & 2012-11-01 & US & $\begin{array}{l}\text { Cray/HPE Cray XK7, AMD Opteron } 6274 \text { 16C @ } \\
2.2 \mathrm{GHz} \text { w/ Cray Gemini interconn., Nvidia K20x GPUs }\end{array}$ & 560640 \\
\hline $\begin{array}{l}\text { Tianhe- } \\
\text { 2A }\end{array}$ & $\begin{array}{l}\text { June } 2013- \\
\text { Nov. } 2015\end{array}$ & China & Intel Xeon, TH Express-2, Matrix 2000 & $\begin{array}{c}4981 \\
760\end{array}$ \\
\hline $\begin{array}{c}\text { Sunway } \\
\text { Taihu- } \\
\text { Light }\end{array}$ & $\begin{array}{l}\text { Jun } 2016- \\
\text { Nov } 2017\end{array}$ & China & Sunway MPP, Sunway SW26010 260C, NRCPC & $\begin{array}{c}10649 \\
600\end{array}$ \\
\hline Summit & $\begin{array}{l}\text { June } 2018- \\
\text { Nov } 2019\end{array}$ & US & IBM AC922 Power9 + Nvidia GV100 GPUs, Mellanox & $\begin{array}{c}2424 \\
492\end{array}$ \\
\hline $\begin{array}{l}\text { Fugaku } \\
\text { SC }\end{array}$ & $\begin{array}{l}\text { June } 2020- \\
\text { Nov. } 2020\end{array}$ & Japan & $\begin{array}{c}\text { Fujitsu/RIKEN ARM-based A64FX 48C @ } 2.2 \mathrm{GHz} \\
\text { w/ Tofu interconnects }\end{array}$ & $\begin{array}{c}7299 \\
072 \\
\text { (Jun) }\end{array}$ \\
\hline & & & & $\begin{array}{c}7630 \\
848 \\
(\mathrm{Nov})\end{array}$ \\
\hline
\end{tabular}

Table 1: Top500 No. 1 systems 2010-20

\section{ARM - from RISC and Embedded systems to Supercomputers}

ARM is a 30-year old UK-based semiconductor IP (Intellectual Property) company that started as Advanced RISC Machines Ltd., a joint venture that included former UK company Acorn Computers, Apple Computers (now Apple Inc.) (Apple to Join Acorn, VLSI in Chip-Making Venture, n.d.), and VLSI Technology (a US company later bought by Phillips for USD 1 billion and now part of the Philips spin-off NXP Semiconductors). ARM was bought in 2016 by the Japanese conglomerate and investing company Softbank Group, which also has a main stake in US Sprint and also has significant T-Mobile stock via the recent merger of Sprint and T-Mobile that was completed in April 2020. In September 2020, NVIDIA announced that it is buying ARM (NVIDIA to Acquire Arm for USD 40 Billion, n.d.), but it may take time to complete since it is running into some licensing issues regarding its subsidiary ARM - China.

Unlike Intel, AMD, and Freescale, ARM does not produce any computer chips, but licenses its architecture designs and product ecosystem to other vendors. It has particularly focused on low-power CPUs and GPUs for mobile and embedded devices, and has over the last several years acquired dozens of smaller companies, such as Falanx, a Norwegian startup that is now known as ARM Norway and is behind their Mali GPU processor.

ARM now offers a wide range of IP design products, including CPUs, GPUs and microcontrollers. ARM CPUs and NPUs (Neural Processing Units) include Cortex-A, Cortex-M, Cortex-R, Neoverse, Ethos, and SecureCore. Mobile companies such as Apple and Samsung often license the designs from ARM to manufacture and integrate them into their own System-on-chip ( $\mathrm{SoC})$ designs with other components such as GPUs (sometimes ARM's Mali) or modem/radio baseband components (for mobile phones). In fact, due 
to the proliferation of mobile and handheld devices such as tablets, ARM was claimed to have $75 \%$ of the world's CPU market in 2005. As the demand for processing power of these devices has increased so has the demand for their CPUs; since 2018, ARM has also offered server-class CPU designs. If NVIDIA's purchase of ARM goes through, it will be very interesting to see how it impacts future ARM designs that are already influenced by AI workloads.

\section{ARM-based Astra}

The first ARM-based supercomputer to appear on the the Top-500 list was Astra at Sandia National Labs, announced at SC18. The HPE (Hewlett Packard Enterprise)-built system ranked at number 204 with 1.529 petaFLOPS, and made number 36 on the HP Conjugate Gradient benchmark, a benchmark more targeted to its use. Astra was the first system which that was part of the US DOE NNSA (National Nuclear Security Administration) Vanguard program that looks at prototype systems for advanced architectures.

One of the Astra nodes is about 100 times faster than the ARM-based CPU chips found in cell phones. The Astra system has 5,184 ARM-based Cavium ThunderX2 28-core processors. Cavium Inc. grew from designing networking processors to itself being bought in 2018 by Marvell Technology group, a Bermudaregistered conglomerate.

Michael Aguilar, the main systems administrator of Astra, gave a nice introduction to the system in 2019 at Stanford (ASTRA: A Large Scale ARM64 HPC Deployment - insideHPC, n.d.). He describes how they have ported many of the familiar packages ranging from OpenMPI to UCX, where one of the interesting challenges they faced was that, unlike Intel cores, the ARM cores did not appear next to each other, so they behaved in a very non-uniform fashion. Socket-direct, a new feature on the architecture, seems to alleviate many performance issues associated with core locality, but requires a new awareness at this level. They have had good results with the system so far, achieving from 1.6X speedup on Monte Carlo to 1.87 on linear solver versus the Intel Haswell-based Trinity ASC platform they used as a baseline.

However, as far as power consumption, they did not see it particularly more efficient than the Intel ones, but have not been focusing on this either.

\section{Fugaku and ARM}

In Summer 2020, the new Japanese Fugaku Supercomputer at RIKEN gained the top spot on the Top-500 list. Named "High Peak" after Mt Fuji, its ARM-based A64FX 48C chips are jointly developed by RIKEN and Fujitsu and uses Fujitsu's Tofu (torus fusion) interconnects. The A64 chip is a TSMC 7nm FinFET with 8.786 billion transistors. And 594 signal pins. It also has SVE 512 x 2 vector extensions (Supercomputer Fugaku: Fujitsu Global, n.d.).

Note that one of the biggest differences between x86 chips from Intel and AMD, from a vector operation point of view, is that modern Intel chips have one 512-bit AVX2 vector unit, whereas AMD chips have 2-3 128-bit units.

Fugaku maintained its number-1 spot in November 2020 (Japan's Fugaku keeps position as fastest supercomputer, n.d.), but had, unlike the 3 next systems (Summit, Sierra, and Sunway), added over 300,000 cores (from 7,299,072 cores to 7,630,848 cores). Its A64 chip has in addition to the 48-core compute unit, 2 or 4 assist units that run the OS (operating system).

The Fugaku system is also very energy efficient, ranking number 10 on the Green500 list, unlike Summit, Sierra, and Sunway. Selene, the Nvidia DGX A100 system at NVIDIA with 555,520 cores, ranked in Nov. 2020 at number 5 both on the Top-500 and Green500 lists. 
The Fujitsu A64fx is also featured in Stony Brook's HPE Apollo 80/ Cray computer Ookami ("wolf" in Japanese), announced in Nov. 2020 . (Cray was formally acquired by HPE in Sept. 2019.) The Ookami system is the first system announced outside Japan that will feature the A64fx.

\section{Atos and AMD EPYC}

Another interesting entry on the Nov. 2020 Top-500 list is the number-7 JUWELS Booster Module, an Atos Bull Sequena XH200 based on the AMD EPYC chips installed at Forschungzentrum Jülich (FZJ) in Germany.

Atos is a French company that emerged from the merger of two French companies and a Dutch IT company. It has since grown with the merger/acquisition of several companies, including Siemens IT (2011), Bull, and Xerox ITO (2014), the latter a well-known HPC vendor in Europe. It has also strengthened its US presence through the acquisition of Xerox ITO (2014) and Syntel (2018).

As a historical note, Heide (Heide, 1991) (Heide, 2008) describes how both Bull and IBM have their roots dating back to punch-card machines. Bull is in fact named after the Norwegian pioneer, Fredrik Rosing Bull who in 1918 started to build punched-card machines. Bull died in 1924, but his work was continued by others in Norway, and later (1931) it was relocated to Paris in France where it, after several reorganizations, emerged as Bull, a subsidiary of Atos Technologies.

In May 2020 Atos announced that it was adding the new NVIDIA A100 GPUs to the Jülich system (Atos Launches First Supercomputer Equipped with NVIDIA A100 GPU - Atos, n.d.), which clearly contributed to its number 7 Nov. 2020 ranking on the Top-500.

\section{The nm Race — Intel versus TSMC}

Currently, just three chip manufacturers in the world are using less than $14 \mathrm{~nm}$ node technologies: Intel, TSMC (Taiwan Semiconductor Manufacturing Company), and Samsung. China's SMIC has yet to do smaller than $14 \mathrm{~nm}$ processes. The $\mathrm{nm}$ (nanometer) denomination used to refer to gate length, but has in the last several years been tied to "technology node" size and become more vendor-dependent. For instance, Intel's $10 \mathrm{~nm}$ nodes are assumed to be compatible with foundries' $7 \mathrm{~nm}$ nodes.

So how is this affecting Supercomputing?

Fugaku and AMD's recent EPYC 7nm chip are both manufactured at TSMC, which also produces the new $5 \mathrm{~nm}$ M1 chip for Apple. However, TSMC reports it is struggling to meet the demand for the Apple chips, so Apple may have to again also use Samsung.

Intel announced through its earnings statement in July 2020 that it is having issues with its $7 \mathrm{~nm}$ manufacturing, thus causing an expected 1-year or more delay of its GPU Ponte Vecchio chips. This may again delay the Aurora system planned at Argonne National Labs (Intel delays 7nm, considers using rival foundries, n.d.) (AMD-Powered Frontier May Become First US Exascale Supercomputer Due to Intel's 7nm Process Issues, n.d.), one of the two new exascale systems planned in the US in 2021. The AMD-powered Frontier system at Oak Ridge National Laboratory is supposedly still on schedule, and will likely become the first exascale system in the US.

Of course, if we allow for low- or mixed-precision and look at the HPL-AI benchmarks - differing from the Linpack benchmarks used for the Top-500 rankings that only allow for full 64-bit precisions - we are already in the exascale era. Fugaku also ranked number 1 on this benchmark in Nov. 2020 peaking at 2.0 exaFLOPS (John Boyd, IEEE Spectrum Tech Talk, June 2020:Full Page Reload, n.d.). 


\section{EuroHPC JU and the next wave of European supercomputers}

Europe have had some systems in the top ten of the Top-500 list, including the current Atos systems, but is now pushing hard for their new EuroHPC JU (European HPC Joint Undertaking) with a roadmap that includes the following systems:

\section{Pre-Exascale class:}

- LUMI (Large Unified Modern Infrastructure) at CSC's center in Kajaani, Finland (LUMI, n.d.) (EuroHPC Exec. Dir. Talks Procurement, EPI, and Europe's Efforts to Control its HPC Destiny, n.d.)

- Marenostrum 5 at Barcelona Supercomputing Centre, Spain

- Leonardo at CINECA, Italy (CINECA Partners with Atos for 240PFLOPS 'Leonardo' Supercomputer - insideHPC, n.d.)

\section{Petascale supercomputers:}

- LuxProvide, Luxembourg

- IZUM, Slovenia

- IT4Innovations National Supercomputing Centre, Czech Republic

- Sofiatech, Bulgaria

- Minho Advanced Computing Centre (MACC), Portugal,

LUMI will be an HPE Cray EX supercomputer with AMD 64-core EPYC CPUs and feature the new AMD Instinct GPUs, and is projected to be faster than the current number 1 Fugaku supercomputer. And Leonardo is now announced to be a 240-petaFLOPS Atos' BullSequana XH2000 with ParTec's ParaStation Modulo software. Marenostrum 5's design has not been announced as of this article's writing.

\section{FPGA - finally maturing for HPC?}

Field-Programmable Gate Arrays (FPGAs) that let you encode algorithms and functions in hardware offer great potential speeds for user application codes, but have been, and still are, fairly hard to program for scientists. However, two of the word's largest CPU makers have bought FPGA companies (AMD announced in October 2020 it will be buying the FPGA company Xilinx Inc.). Although Xilinx is headquartered in San Jose, it has a strong presence in Europe and Asia, and has also started to offer FPGA-based accelerator cards targeting AI and cloud workloads. The deal is expected to go through by the end of 2021. Xilinx new data center accelerator card, the Alveo FPGA U250, targets AI/ML, graphics computing and HPC computations (Alveo U250 Data Center Accelerator Card, n.d.).

FPGAs and other emerging Accelerators will be discussed in a future department feature.

\section{References}

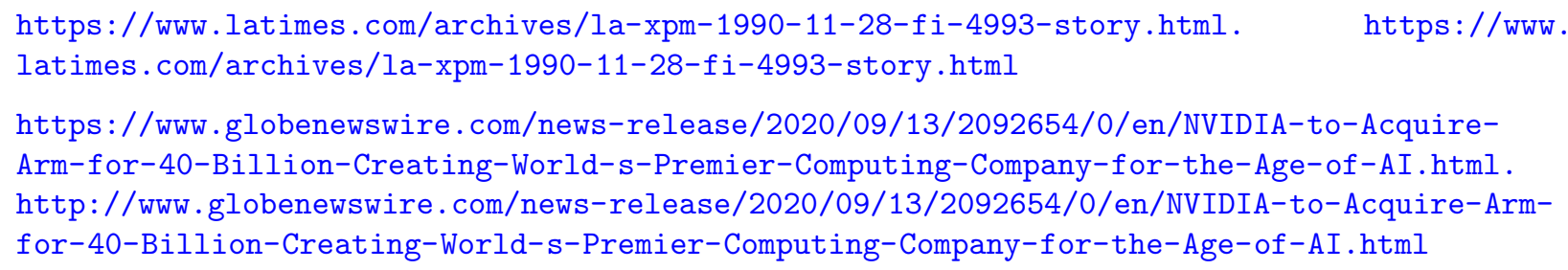


https://insidehpc.com/2019/02/astra-a-large-scale-arm64-hpc-deployment/.

https :

//insidehpc.com/2019/02/astra-a-large-scale-arm64-hpc-deployment/

https://www.fujitsu.com/global/about/innovation/fugaku/. https://www.fujitsu.com/global/ about/innovation/fugaku/

https: //www.japantimes.co.jp/news/2020/11/17/national/japan-fugaku-fastest-supercomputer/ - https://www.japantimes.co.jp/news/2020/11/17/national/japan-fugaku-fastestsupercomputer/

From Invention to Production: The Development of Punched-card Machines by F. R. Bull and K. A. Knutsen 1918-1930. (1991). IEEE Annals of the History of Computing, 13(3), 261-272. https://doi.org/10.1109/ mahc. 1991.10024

Punched cards for professional European offices: revisiting the dynamics of information technology diffusion from the United States to Europe 1889-1918. (2008). History and Technology, 24(4), 307-320. https: //doi.org/10.1080/07341510802044686

https://atos.net/en/2020/press-release/general-press-releases_2020_05_14/atos-launchesfirst-supercomputer-equipped-with-nvidia-a100-gpu. https://atos.net/en/2020/pressrelease/general-press-releases_2020_05_14/atos-launches-first-supercomputer-equippedwith-nvidia-a100-gpu

https ://www .datacenterdynamics . com/en/news/intel-delays-7nm-considers-using-rivalfoundries/. https://www.datacenterdynamics.com/en/news/intel-delays-7nm-considers-usingrival-foundries/

https://phonemantra.com/amd-powered-frontier-may-become-first-us-exascale-supercomputerdue-to-intels-7nm-process-issues/. https://phonemantra.com/amd-powered-frontier-maybecome-first-us-exascale-supercomputer-due-to-intels-7nm-process-issues/

https://spectrum.ieee.org/tech-talk/computing/hardware/japans-fugaku-supercomputeris-first-in-the-world-to-simultaneously-top-all-high-performance-benchmarks. https: //spectrum. ieee.org/tech-talk/computing/hardware/japans-fugaku-supercomputer-is-firstin-the-world-to-simultaneously-top-all-high-performance-benchmarks

https://www.lumi-supercomputer.eu/lumi-one-of-the-worlds-mightiest-supercomputers/value here

https://www.hpcwire.com/2020/11/19/eurohpc-exec-dir-talks-procurement-epi-and-europesefforts-to-control-its-hpc-destiny/value here

https://insidehpc.com/2020/11/cineca-partners-with-atos-for-240pflops-leonardosupercomputer/. https://insidehpc.com/2020/11/cineca-partners-with-atos-for-240pflopsleonardo-supercomputer/

https://www.xilinx.com/products/boards-and-kits/alveo/u250.html. https://www.xilinx.com/ products/boards-and-kits/alveo/u250.html 